\title{
Gold Nanorods: Near-Infrared Plasmonic Photothermal Conversion and Surface Coating
}

\author{
Bo Cong ${ }^{1}$, Caixia Kan ${ }^{1,2 *}$, Honggeng Wang ${ }^{1}$, Jinsheng Liu ${ }^{1}$, Haiying $\mathrm{Xu}^{1}$, Shanlin $\mathrm{Ke}^{1}$ \\ ${ }^{1}$ College of Science, Nanjing University of Aeronautics and Astronautics, Nanjing, China \\ ${ }^{2}$ Key Laboratory for Intelligent Nano Materials and Devices of the Ministry of Education, China \\ Email: congbobob@hotmail.com, ${ }^{*}$ cxkan@nuaa.edu.cn
}

Received October 2013

\begin{abstract}
In this paper, AuNRs colloids with $\mathrm{SPR}_{\mathrm{L}}$ located at $\sim 810 \mathrm{~nm}$ and $\sim 1100 \mathrm{~nm}$ were synthesized using an improved seed method. Based on the NIR lasers available, photothermal conversion of AuNRs were systematically studied compared with that of water. Under low power irradiation, the highest temperature is obtained when the SPR wavelength of AuNRs is equal to the laser wavelength, and temperature of colloid increases from $\sim 20^{\circ} \mathrm{C}$ to $\sim 65^{\circ} \mathrm{C}$. With increasing laser power (such as $6 \mathrm{~W}$ ), the AuNRs colloid boils within a few minutes, and nanorods undergo a shape deformation from rod to spherical particle and even fusion, and the $S P R_{L}$ disappears. For further investigation, the obtained AuNRs were coated with $\mathrm{SiO}_{2}$ shell to form a core-shell nanostructure $\left(\mathrm{Au} @ \mathrm{SiO}_{2}\right)$. The surface coating can be used not only in keeping the stability of AuNRs for further treatment, but also in increasing plasmonic property and biocompatibility. This work will be useful for designing plasmonic photothermal properties and further applications in nanomedicine.
\end{abstract}

\section{KEYWORDS}

Gold Nanorods; Surface Plasmon Resonance; Photothermal Effect; Core-Shell

\section{Introduction}

Gold nanorod is one of the most studied colloidal nanostructures for its two distinct surface plasma resonances (SPR), known as the transverse mode $\left(\mathrm{SPR}_{\mathrm{T}}\right)$ and longitudinal mode $\left(\mathrm{SPR}_{\mathrm{L}}\right)$ [1-4]. The $\mathrm{SPR}_{\mathrm{T}}$ is weak and located at $\sim 520 \mathrm{~nm}$, while the strong $\mathrm{SPR}_{\mathrm{L}}$ can be tuned in the visible-near infrared (Vis-NIR) region [5-7]. Wet chemical fabrication of AuNRs was pioneered by Murphy and coworkers through a citrate-capped seed technique $[8,9]$. Improvement of this method for producing AuNRs with high yield was achieved by introduction of CTAB [10-12].

The optical tunability of AuNRs has brought several advantages to AuNRs. For example, using the tunability (and sensitivity) of SPR to aspect ratios of AuNRs and polarization of light, five-dimensional optical recordingreading process was realized [13]. The changes of density of surface charge and surrounding index can be used for the direct observation of chemical reactions $[14,15]$. The high dependence of $\mathrm{SPR}_{\mathrm{L}}$ on aspect ratios, surface coating, and aggregation degree or assembly is promising for chemical and biological sensing. The NIR $\mathrm{SPR}_{\mathrm{L}} \mathrm{ex}-$

"Corresponding author. tinction is consistent with the human tissue facilitating photothermal therapy and solar energy harvesting [1619]. For in-vivo therapy in cancer research, small diameter AuNRs are more ideal metal nanostructures for cancer diagnosis and therapy for their tunable $\mathrm{SPR}_{\mathrm{L}}$ and optimal penetration through biological skin and tissue in the NIR region [20-25]. And the photothermal conversion efficiency is an essential parameter in the therapy $[26,27]$.

However, due to the toxicity of CTAB covered on AuNRs, surface coupling with biological compatibility molecular was usually proposed for application research [28]. On the other hand, surface coating with medium to form a core-shell structure also provides an effective way to keep the stability in further treatment. Silica $\left(\mathrm{SiO}_{2}\right)$, as a gain medium for plasmonic property and advantage of biological compatibility and chemical stability, was a promising coating medium, especially for surface coating of AuNRs [29]. The $\mathrm{SiO}_{2}$ coating can be used not only in keeping the stability of AuNRs for further treatment, but also in increasing plasmonic property and biocompatibility.

In this work, AuNRs colloids with $\mathrm{SPR}_{\mathrm{L}}$ absorption peaks were synthesized using an improved "seed" method. Based on the NIR lasers available, such as 808 
nm and $1064 \mathrm{~nm}$, the photothermal conversion of AuNRs colloids was studied under different irradiation performance. [30] Under laser radiation, temperatures of AuNRs colloids rise obviously. With increasing the laser power (such as $6 \mathrm{~W}$ ), the AuNRs colloid boils within a few minutes, and nanorods undergo a shape deformation from rod to spherical particle and even fusion. For further research, $\mathrm{SiO}_{2}$ coating on AuNRs to form $\mathrm{Au} @ \mathrm{SiO}_{2}$ core-shell structure was also carried out.

\section{Experiment Section}

\subsection{Synthesis of AuNRs}

AuNRs colloids with desired $\mathrm{SPR}_{\mathrm{L}}$ wavelengths (such as $810 \mathrm{~nm}$ and $1100 \mathrm{~nm}$ ) can readily be synthesized through a similar process, as reported previously [31,32]. Briefly, in the first step, the seed solution for AuNRs was prepared by dissolving $\mathrm{HAuCl}_{4}(0.05 \mathrm{~mL}, 0.05 \mathrm{M})$ in an aqueous solution of CTAB $(10 \mathrm{~mL}, 0.1 \mathrm{M})$. Then, icecold $\mathrm{NaBH}_{4}(0.6 \mathrm{~mL}, 0.01 \mathrm{M})$ was injected into the solution under vigorous stirring. In the second step of the growth solution for GNRs with $\mathrm{SPR}_{\mathrm{L}}$ at $805 \mathrm{~nm}, 0.912 \mathrm{~g}$ CTAB together with 0.110 g 5-bromosalicylic acid was dissolved in $49 \mathrm{~mL}$ water. Then $\mathrm{AgNO}_{3}$ (0.24 mL - $1 \mathrm{~mL}$, $0.02 \mathrm{M}$ ) was added under stirring, followed by addition of $\mathrm{HAuCl}_{4}(0.5 \mathrm{~mL}, 0.05 \mathrm{M})$. Then $0.13 \mathrm{~mL}(0.1 \mathrm{M})$ AA solution was added with stirring until the solution became colorless. After aging $30 \mathrm{~min}, 0.06 \mathrm{~mL}$ "seed" solution was added into the as-prepared growth solution, and AuNRs can be obtained after $12 \mathrm{~h}$.

\subsection{Photothermal Conversion of AuNRs}

The setup for the measuring the photothermal conversion was composed of a $10 \mathrm{~mm}$ path length quartz cuvette, a sensitive digital thermometer (TM-902C), and continuous lasers (including $808 \mathrm{~nm}$ laser coupled out through an optical fiber, GKFCM-808; semiconductor diode 980 nm laser; and 1064 nm, GKNQL-1064). The laser power was obtained from the P-I curves. The laser light illuminated on the AuNRs colloid in the cuvette. After each irradiation, the probe head of the thermocouple was completely submerged in the colloid immediately. The temperature of the colloid is reasonably assumed to be uniform in the solution for the same testing data at different positions.

\subsection{Surface Silica Coating AuNRs}

$0.04 \mathrm{~mL} \mathrm{NaOH}(0.1 \mathrm{M})$ solution was added into the as-obtained Au NRs colloid (4 mL) with a certain CTAB concentration under stirring. Then $0.04 \mathrm{~mL}$ TEOS (in ethanol) was injected into Au NRs colloid under gentle rotary shaking for the hydrolysis of TEOS and $\mathrm{SiO}_{2}$ coating on the surface of Au NRs.Au@SiO ${ }_{2}$ core-shell structure was obtained after $20 \mathrm{~h}$.

\subsection{Characterization}

UV-Vis-NIR spectra of prepared solution were collected by spectrophotometer (UV-6300) in the wavelength range of $200-1100 \mathrm{~nm}$. The products were purified by first centrifugating at $3000 \mathrm{RPM}$ for $20 \mathrm{~min}$ to remove the large sized particles deposited on the bottom of centrifugal tube. Then the colloids were centrifugated repeatedly at 14000 RPM with deionized water. Then the samples were deposited on copper grids covered by an amorphous carbon film for transmission electron microscope (TEM: JEOL-100CX) measurements.

\section{Results and Discussion}

The microstructure and growth mechanism of the AuNRs were studied in detail, as reported previously [31]. Fig. 1a shows the normalized UV-Vis-NIR absorption spectra for the AuNRs colloids. In addition to the strong and tunable $\mathrm{SPR}_{\mathrm{L}}$ absorption peak located at $\sim 810$ and 1100 $\mathrm{nm}$, respectively, there is a weak resonance peak located at $\sim 520 \mathrm{~nm}$, originated from the $\mathrm{SPR}_{\mathrm{T}}$ absorption of nanorods and possible existence of spherical nanoparticles and nanocubes. TEM images of the AuNRs with $\mathrm{SPR}_{\mathrm{L}}$ located at $\sim 810 \mathrm{~nm}$, and $\sim 1100 \mathrm{~nm}$ are shown in Figures 1(b) and (c). The sizes of the AuNRs are nearly uniform.

The effects of the SPR wavelength and NIR laser on the photothermal conversion efficiencies of AuNRs are studied by measuring the temperature increase, compared with that of water. Under low power irradiation, it is shown that the highest temperature is obtained when the $\mathrm{SPR}_{\mathrm{L}}$ wavelength of AuNRs is equal to the illumination laser wavelength, and temperature of the colloid rises from $\sim 20^{\circ} \mathrm{C}$ to $\sim 65^{\circ} \mathrm{C}$, which is accordant with the reported work [32]. However, the photothermal efficiency
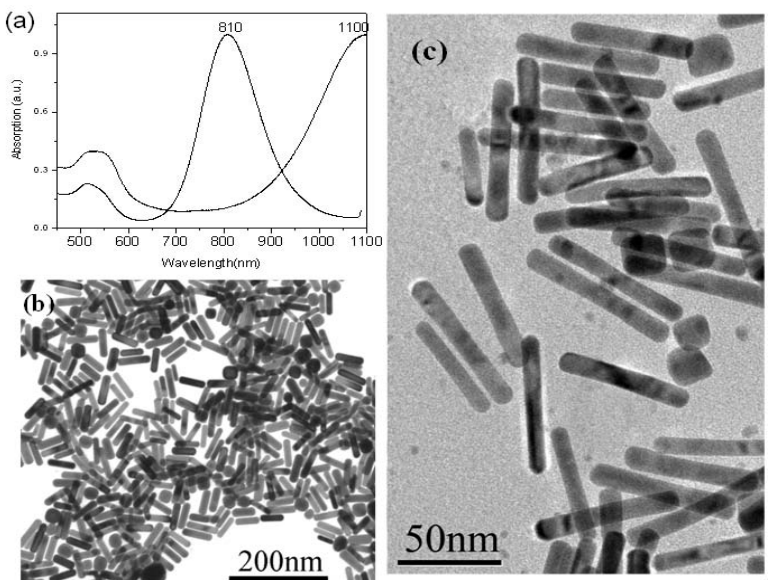

Figure 1. UV-Vis-NIR absorption spectra (a) and TEM images for the as-prepared AuNRs colloids with $S_{P R}$ absorption located at $\sim 810$ (b) and $\sim 1100 \mathrm{~nm}$ (c). 
decreased with increasing irradiating time. As shown in Tables 1 and $\mathbf{2}$ for the photothermal effect of AuNRs colloid $(1 \mathrm{~mL})$ with $\mathrm{SPR}_{\mathrm{L}}$ peaks located at $\sim 805$ and $1100 \mathrm{~nm}$ irradiated, respectively, under 808 and $1064 \mathrm{~nm}$ lasers. Since AuNRs were dispersed in deionized water, we used the heat capacity of water $4.2 \mathrm{~J} / \mathrm{g}$ in calculating the efficiency of AuNRs colloid.

Figures 2(a) and (b) showed the optical absorption spectra of AuNRs colloids before and after irradiation with high laser power. After the AuNRs colloid with $\mathrm{SPR}_{\mathrm{L}}$ at $810 \mathrm{~nm}$ was irradiated under $808 \mathrm{~nm}$ laser $(6 \mathrm{~W})$ for $3 \mathrm{~min}$, the intensity of $\mathrm{SPR}_{\mathrm{L}}$ decreased sharply, and the absorption damped over the whole region. The $\mathrm{SPR}_{\mathrm{L}}$ at $1100 \mathrm{~nm}$ disappeared after the AuNRs colloid was irradiated by $1064 \mathrm{~nm}$ laser (7 W) for $8 \mathrm{~min}$, and only the absorption peak of gold spherical nanoparticles was observed, as inserted one in Figure 2(b).
With increasing the laser power (such as 6W for 808 $\mathrm{nm}$ laser), the AuNRs colloid would boil within a few minutes. After the strong irradiation, the color of the colloid faded with products deposited on the bottom. Corresponding to the optical spectra changes of the AuNRs colloid under strong laser irradiation, TEM images indicated that nanorods undergo deformation from rod to spherical particle and even fusion under irradiation of high power. Figures 3(a), (b) presents the TEM images of the AuNRs (with $\mathrm{SPR}_{\mathrm{L}}$ at $1100 \mathrm{~nm}$ ) before and after laser irradiations. After the AuNRs colloid was irradiated with $1064 \mathrm{~nm}$ laser (7 W) for $8 \mathrm{~min}$, the aspect ratio decreased and large sized nanoparticles were observed. The morphology evolution of the AuNRs under high power laser irradiation can be used to explain the disappearance of $\mathrm{SPR}_{\mathrm{L}}$ and the photothermal efficiency decrease of AuNRs colloid with increasing irradiating time.

Table 1. Photo-thermal effect of AuNRs colloid (1 mL) with $S_{P R}$ peaks located at $\sim 810 \mathrm{~nm}$ under $808 \mathrm{~nm}$ lasers (laser spot size: $\mathbf{2} \mathrm{mm}$ ) irradiation. (In calculating the efficiency of AuNRs colloid, the heat capacity of water is $4.2 \mathrm{~J} / \mathrm{g}$ ).

\begin{tabular}{cccccc}
\hline $\begin{array}{c}\text { Power } \\
(\mathrm{W})\end{array}$ & $\begin{array}{c}\text { Irradiation time } \\
(\mathrm{min})\end{array}$ & $\begin{array}{c}\mathrm{T}\left({ }^{\circ} \mathrm{C}\right) \text { of sample before and } \\
\text { after irradiation }\end{array}$ & $\begin{array}{c}\text { Efficiency of AuNRs } \\
\text { colloid }\end{array}$ & $\begin{array}{c}\mathrm{T}\left({ }^{\circ} \mathrm{C}\right) \text { of pure water before and } \\
\text { after irradiation }\end{array}$ & $\begin{array}{c}\text { Efficiency } \\
\text { of pure water }\end{array}$ \\
\hline & 2 & $22 / 27$ & 0.525 & $18 / 18$ & 0 \\
0.333 & 5 & $22 / 31$ & 0.372 & $18 / 18$ & 0.021 \\
& 10 & $22 / 35$ & 0.269 & $18 / 19$ & 0.0525 \\
0.667 & 2 & $22 / 35$ & 0.682 & $19 / 20$ & 0.063 \\
& 5 & $22 / 46$ & 0.503 & $19 / 23$ & 0.042 \\
& 10 & $22 / 52$ & 0.315 & $20 / 21$ & 0.0438 \\
& 2 & $22 / 36$ & 0.612 & $20 / 24$ & 0.07 \\
\end{tabular}

Table 2. Photo-thermal effect of AuNRs colloid (1 mL) with $S P R_{L}$ peaks located at $1100 \mathrm{~nm}$ under $1064 \mathrm{~nm}$ lasers (laser spot size: $\mathrm{r}=1 \mathrm{~mm}$ ) irradiation.

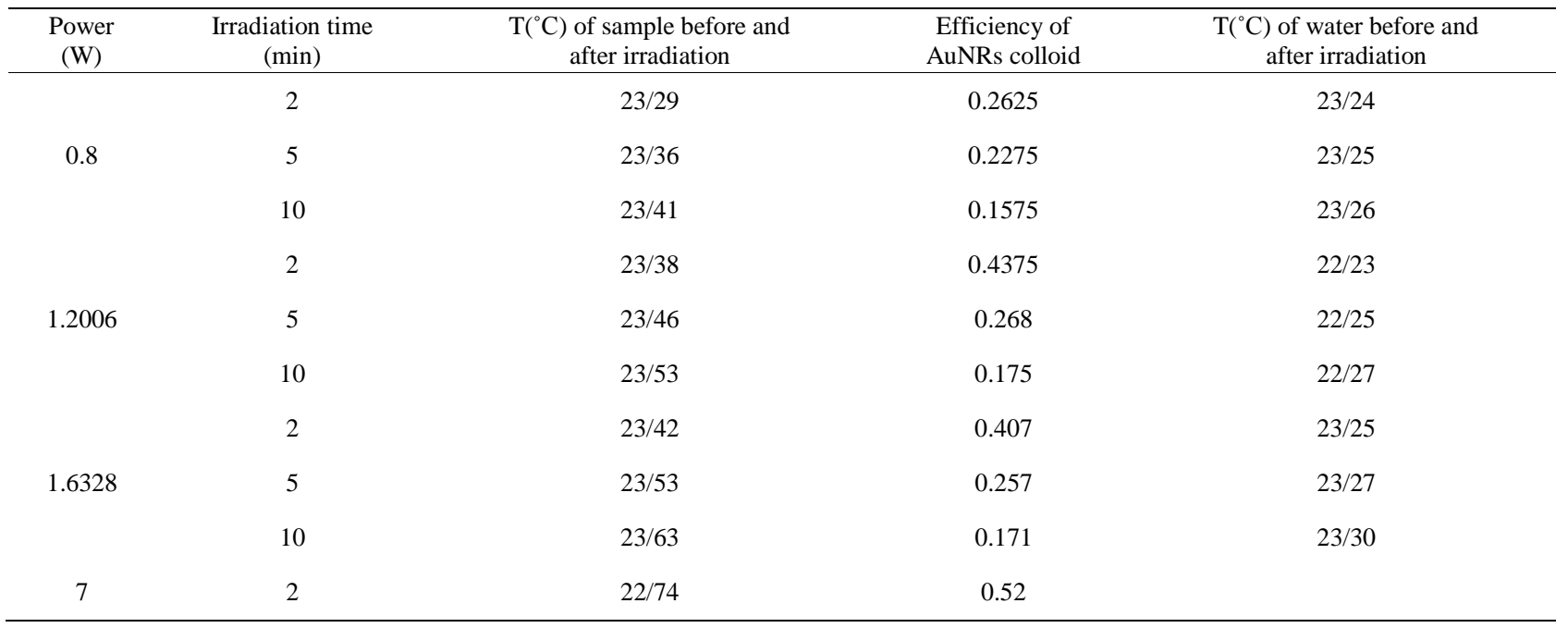



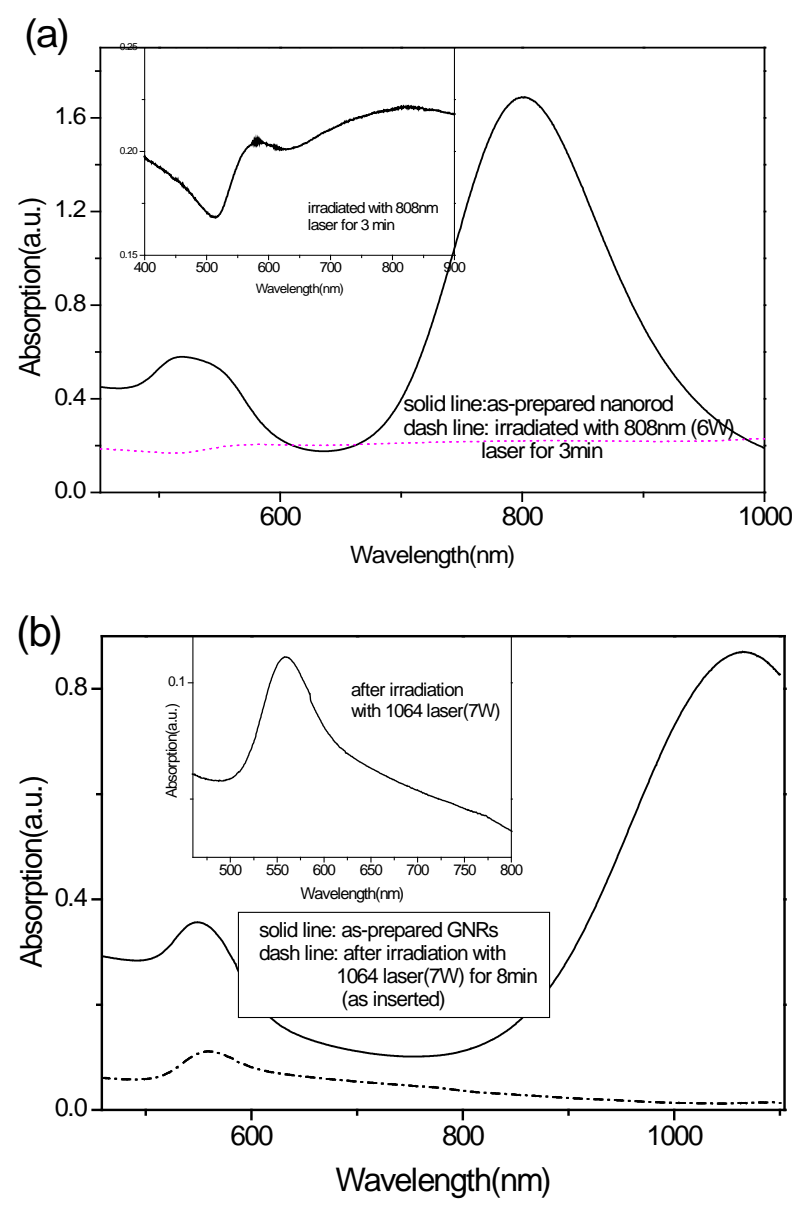

Figure 2. UV-Vis-NIR absorption spectra of AuNRs colloids before and after irradiation with high laser power, insets are the spectra of AuNRs after irradiation. AuNRs colloids with SPRL located at $\sim 810 \mathrm{~nm}$ (a) and $1100 \mathrm{~nm}$ (b) before and after irradiation under $808 \mathrm{~nm}$ laser $(6 \mathrm{~W}, 3 \mathrm{~min}$ ) and $1064 \mathrm{~nm}$ laser (7 W, 2 min), respectively.
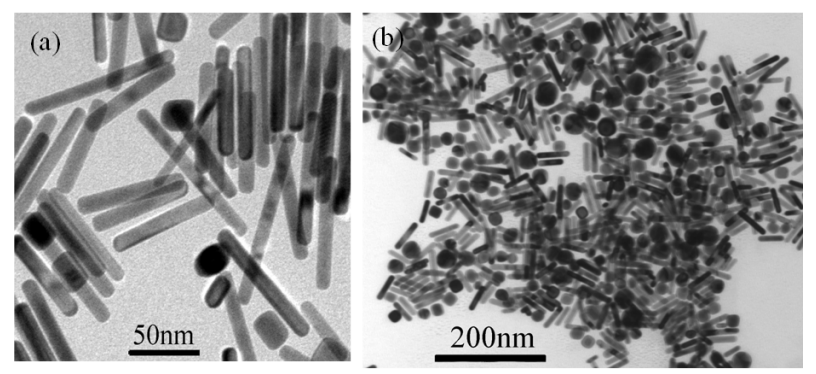

Figure 3. (a), (b) TEM images of the AuNRs (with SPRL at $1100 \mathrm{~nm}$ ) before and after irradiations under $1064 \mathrm{~nm}$ laser (7 W) for $8 \mathrm{~min}$.

Figure 4(a) presents the TEM image of AuNRs after $\mathrm{SiO}_{2}$ coating. It can be seen that most of the AuNRs, together with some $\mathrm{Au}$ nanoparticles, have been successfully sealed by homogeneous $\mathrm{SiO}_{2}$, and formed coreshell Au@SiO 2 nanostructure. Also, there are a few $\mathrm{SiO}_{2}$ nanospheres without AuNRs core. By controlling the

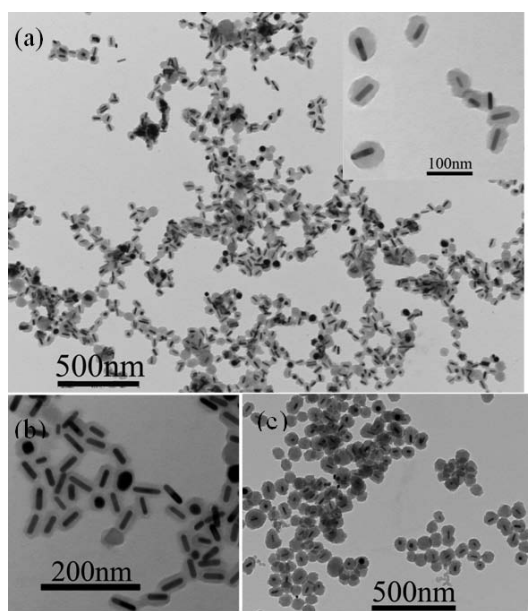

Figure 4. (a)TEM images of silica-coated AuNRs. Insertion of (a) is the magnified image of core-shell structured Au@SiO . (b-c) TEM images of AuNRs coated with silica shells of different thickness.

coating time, volume of TEOS/ethanol, and the concentration of AuNRs, we were able to vary the shell thickness (Figures 4(b) and (c)). In the experiment, it is found that certain CTAB coverage on the surface of AuNRs is very important for the $\mathrm{SiO}_{2}$ capping. And further experiments are now being carried out.

\section{Conclusion}

In summary, we have demonstrated the synthesis of AuNRs with $\mathrm{SPR}_{\mathrm{L}}$ in the NIR region. The photothermal conversion efficiencies of AuNRs were studied. An obvious temperature increasing was observed in the photothermal conversions of AuNRs under laser irradiation. The highest temperature is obtained when the $\mathrm{SPR}_{\mathrm{L}}$ wavelength of AuNRs is equal to the laser wavelength, and temperature of the colloid increased up to $\sim 65{ }^{\circ} \mathrm{C}$ even at low irradiating power. At a relative high power (such as $6 \mathrm{~W}$ ), nanorods undergo deformation from rod to spherical particle and even fusion $\mathrm{Au} @ \mathrm{SiO}_{2}$ core-shell structure was obtained, and further comparing experiments, such as photothermal conversions and stability are now being carried out.

\section{Acknowledgements}

This study was financially supported by National Natural Science Foundation of China (Nos. 11274173, 51032002, 61222403).

\section{REFERENCES}

[1] H. Ma, P. M. Bendix and L. B. Oddershede, "Large-Scale Orientation Dependent Heating from a Single Irradiated Gold Nanorod,” Nano Letters, Vol. 12, No. 8, 2012, pp. 3954-3960. http://dx.doi.org/10.1021/nl3010918 
[2] C. Kuemin, L. Nowack, L. Bozano, N. D. Spencer and H. Wolf, "Oriented Assembly of Gold Nanorods on the Single-Particle Level,” Advanced Functional Materials, Vol. 22, No. 4, 2012, pp. 702-708. http://dx.doi.org/10.1002/adfm.201101760

[3] S. E. Lohse and C. J. Murphy, "The Quest for Shape Control: A History of Gold Nanorod Synthesis," Chemistry of Materials, Vol. 25, No. 8, 2013, pp. 1250-1261. http://dx.doi.org/10.1021/cm303708p

[4] M. Quinten, "Optical Properties of Nanoparticle Systems: Mie and Beyond,” 1st Edition, Wiley-VCH Verlag GmbH \& Co. KGaA, Weinheim, 2011.

[5] L. Vigderman, B. P. Khanal and E. R. Zubarev, "Functional Au Nanorods: Synthesis, Self-Assembly and Sensing Applications,” Advanced Materials, Vol. 24, No. 36, 2012, pp. 4811-4841. http://dx.doi.org/10.1002/adma.201201690

[6] D. Nepal, K. Park and R. A. Vaia, "High-yield Assembly of Soluble and Stable Gold Nanorod Pairs for High-Temperature Plasmonics,” Small, Vol. 8, No. 7, 2012, pp. 1013-1020. http://dx.doi.org/10.1002/smll.201102152

[7] L. B. Zhang, X. Zhou, S. X. Bao, Y. F. Shi, Y. Wang, "Rational Design and SERS Properties of Side-by-Side, End-to-End and End-to-Side Assemblies of Au Nanorods," Journal of Materials Chemistry, Vol. 21, 2011, pp. 14448-14455. http://dx.doi.org/10.1039/C1JM11193K

[8] N. R. Jana, L. Gearheart and C. J. Murphy, "Wet Chemical Synthesis of High Aspect Ratio Cylindrical Au Nanorods,” The Journal of Physical Chemistry B, Vol. 105, No. 19, 2001, pp. 4065-4067. http://dx.doi.org/10.1021/jp0107964

[9] N. R. Jana, L. Gearheart and C. J. Murphy, "Seed-Mediated Growth Approach for Shape-Controlled Synthesis of Spheroidal and Rod-Like Gold Nanoparticles Using a Surfactant Template,” Advanced Materials, Vol. 13, No. 18, 2001, pp. 1389-1393. http://dx.doi.org/10.1002/1521-4095(200109)13:18<1389 $\because$ AID-ADMA1389>3.0.CO;2-F

[10] B. Nikoobakht and M. A. El-Sayed, "Preparation and Growth Mechanism of Gold Nanorods (NRs) Using Seed-Mediated Growth Method," Chemistry of Materials, Vol. 15, No. 10, 2003, pp. 1957-1962. http://dx.doi.org/10.1021/cm020732l

[11] K. C. Woo, L. Shao, H. J. Chen, Y. Liang and J. F. Wang, "Universal Scaling and Fano Resonance in the Plasmon Coupling between Gold Nanorods," ACS NANO, Vol. 5, No. 7, 2011, pp. 5976-5986. http://dx.doi.org/10.1021/nn2017588

[12] L. Shao, K. C. Woo, H. J. Chen, Z. Jin and J. F. Wang, "Angle- and Energy-Resolved Plasmon Coupling in Gold Nanorod Dimers,” ACS NANO, Vol. 4, No. 6, 2010, pp. 3053-3062. http://dx.doi.org/10.1021/nn100180d

[13] P. Zijlstra, J. W. M. Chon and M. Gu, "Five-Dimensional Optical Recording Mediated by Surface Plasmons in Gold Nanorods,” Nature, Vol. 459, 2009, pp. 410-413. http://dx.doi.org/10.1038/nature08053

[14] C. Novo, A. M. Funston and P. Mulvaney, "Direct Obseration of Chemical Reactions on Single Gold Nanocrys- tals Using Surface Plasmon Spectroscopy," Nature Nanotechnology, Vol. 3, 2008, pp. 598-602. http://dx.doi.org/10.1038/nnano.2008.246

[15] E. M. Larsson, C. Langhammer, I. Zoric and B. Kasemo, "Nanoplasmonic Probes of Catalytic Reactions," Science, Vol. 326, No. 5956, 2009, pp. 1091-1094. http://dx.doi.org/10.1126/science.1176593

[16] X. Xu, T. H. Gibbons and M. B. Cortie, "Spectrally -Selective Gold Nanorod Coatings for Window Glass," Gold Bulletin, Vol. 39, No. 4, 2006, pp. 156-165. http://dx.doi.org/10.1007/BF03215549

[17] D. P. Yang and D. X. Cui, "Advances and prospects of Au nanorods," Chemistry-An Asian Journal, Vol. 3, No. 12, 2008, pp. 2010-2022. http://dx.doi.org/10.1002/asia.200800195

[18] X. H. Huang, S. Neretina and M. A. El-Sayed, "Gold Nanorods: From Synthesis and Properties to Biological and Biomedical Applications,” Advanced Materials, Vol. 21, No. 48, 2009, pp. 4880-4910. http://dx.doi.org/10.1002/adma.200802789

[19] R. B. Jiang, S. Cheng, L. Shao, Q. F. Ruan and J. F. Wang, "Mass-Based Photothermal Comparison among Gold Nanocrystals, PbS Nanocrystals, Organic Dyes and Carbon Black," The Journal of Physical Chemistry C, Vol. 117, No. 17, 2013, pp. 8909-8915. http://dx.doi.org/10.1021/jp400770x

[20] H. Wang, T. B. Huff, D. A. Zweifel, W. He, P. S. Low, "In Vitro and In Vivo Two-Photon Luminescence Imaging of Single Gold Nanorods," Proceedings of the National Academy of the Sciences of the U.S.A., Vol. 102, No. 44, 2005, pp. 15752-15756. http://dx.doi.org/10.1073/pnas.0504892102

[21] E. B. Dickerson, E. C. Dreaden, X. Huang , I. H. ElSayed and H. Chu, "Gold Nanorod Assisted Near- Infrared Plasmonic Photothermal Therapy (PPTT) of Squamous cell Carcinoma in Mice,” Cancer Letters, Vol. 269, No. 1, 2008, pp. 57-66. http://dx.doi.org/10.1016/j.canlet.2008.04.026

[22] G. von. Maltzahn, J. H. Park, A. Agrawal, N. K. Bandaru, S. K. Das, "Computationally Guided Photothermal Tumor Therapy Using Long-Circulating Gold Nanorod Antennas,” Cancer Research, Vol. 69, 2009, pp. 3892-3900. http://dx.doi.org/10.1158/0008-5472.CAN-08-4242

[23] R. Nadejda and J. Z. Zhang, "Photothermal Ablation Therapy for Cancer Based on Metal Nanostructures," Science in China Series B-Chemistry, Vol. 52, No. 10, 2009, pp. 1559-1575. http://dx.doi.org/10.1007/s11426-009-0247-0

[24] J. Wang, G. Z. Zhu, M. X. You, E. Q. Song and M. I. Shukoor, "Assembly of Aptamer Switch Probes and Photosensitizer on Gold Nanorods for Targeted Photothermal and Photodynamic Cancer Therapy,” ACS NANO, Vol. 6, No. 6, 2012, pp. 5070-5077. http://dx.doi.org/10.1021/nn300694v

[25] Z. Wang, "Plasmon-Resonant Gold Nanoparticles for Cancer Optical Imaging,” Science China Physics, Mechanics \& Astronomy, Vol. 56, No. 3, 2013, pp. 506-513. http://dx.doi.org/10.1007/s11433-013-5006-8 
[26] Z. J. Zhang, L. M. Wang, J. Wang, X. M. Jiang and X. H. Li, "Mesoporous Silica-Coated Gold Nanorods as a Light -Mediated Multifunctional Theranostic Platform for Cancer Treatment,” Advanced Materials, Vol. 24, No. 11, 2012, pp. 1418-1423.

http://dx.doi.org/10.1002/adma.201104714

[27] J. H. Wang, B. Wang, Q. Liu, Q. Li and H. Huang, "Bimodal Optical Diagnostics of Oral Cancer Based on Rose Bengal Conjugated Gold Nanorod Platform,” Biomaterials, Vol. 34, No. 17, 2013, pp. 4274-4283. http://dx.doi.org/10.1016/j.biomaterials.2013.02.012

[28] A. K. Oyelere, P. C. Chen and X. H. Huang, "PeptideConjugated Gold Nanorods for Nuclear Targeting," Bioconjugate Chemistry, Vol. 18, No. 5, 2007, pp. 14901497. http://dx.doi.org/10.1021/bc070132i

[29] T. Ming, L. Zhao, M. Xiao and J. F. Wang, "Resonance-
Coupling-Based Plasmonic Switches,” Small, Vol. 6, No. 22, 2010, pp. 2514-2519. http://dx.doi.org/10.1002/smll.201000920

[30] S. L Ke, C. X. Kan, J. S. Liu and B. Cong, "Controlled Assembly of Gold Nanorods Using Tetrahydrofuran,” RSC Advances, Vol. 3, No. 8, 2013, pp. 2690-2696. http://dx.doi.org/10.1039/C2RA23300B

[31] H. C. Li, C. X. Kan, Z. G. Yi, X. L. Ding and Y. L. Cao, "Synthesis of One Dimensional Gold Nanostructures," Journal of Nanomaterials, Vol. 2010, 2010. http://dx.doi.org/10.1155/2010/962718

[32] H. J. Chen, L. Shao, T. Ming, Z. H. Sun and C. M. Zhao, "Understanding the Photothermal Conversion Efficiency of Gold Nanocrystals," Small, Vol. 6, No. 20, 2010, pp. 2272-2280. http://dx.doi.org/10.1002/smll.201001109 